\title{
Simultaneous identification of the Anopheles funestus group and Anopheles longipalpis type C by PCR-RFLP
}

\author{
Kwang Shik Choi ${ }^{1,2}$, Maureen Coetzee ${ }^{1,2^{*}}$, Lizette L Koekemoer ${ }^{1,2}$
}

\begin{abstract}
Background: Anopheles longipalpis is morphologically similar to the major African malaria vector Anopheles funestus at the adult stage although it is very different at the larval stage. Despite the development of the species-specific multiplex PCR assay for the An. funestus group, the genomic DNA of Anopheles longipalpis type $C$ specimens can be amplified with the Anopheles vaneedeni and Anopheles parensis primers from this assay. The standard, speciesspecific An. funestus group PCR, results in the amplification of two fragments when An. longipalpis type $C$ specimens are included in the analysis. This result can easily be misinterpreted as being a hybrid between An. vaneedeni and An. parensis. Anopheles longipalpis type $C$ can be identified using a species-specific PCR assay but this assay is not reliable if other members of the An. funestus group, such as An. funestus, An. funestus-like and An. parensis, are included. The present study provides a multiplex assay that will identify An. longipalpis along with other common members of the African An. funestus group, including Anopheles leesoni.

Methods: A total of 70 specimens from six species (An. funestus, An. funestus-like, An. parensis, Anopheles rivulorum, An. vaneedeni and An. leesoni) in the An. funestus group and An. longipalpis type C from Malawi, Mozambique, South Africa and Zambia were used for the study. A restriction fragment length polymorphism (RFLP) assay was designed based on the DNA sequence information in the GenBank database.

Results: The enzyme, EcoRl digested only An. longipalpis type $C$ and An. funestus-like after the species-specific An. funestus group PCR assay. The An. longipalpis and An. funestus-like digestion profiles were characterized by three fragments, 376 bp, 252 bp and 211 bp for An. longipalpis type C and two fragments, 375 bp and 15 bp for An. funestus-like.

Conclusions: An RFLP method for the group was developed that is more accurate and efficient than those used before. Hence, this assay would be useful for field-collected adult specimens to be identified routinely in malaria vector research and control studies.
\end{abstract}

\section{Background}

The Anopheles funestus group originally consisted of nine species [1,2]: the major African malaria vector An. funestus and eight minor or non-vectors, Anopheles aruni, Anopheles parensis, Anopheles vaneedeni, Anopheles confusus, Anopheles rivulorum, Anopheles leesoni, Anopheles brucei and Anopheles fuscivenosus. Subsequent studies on the systematics of the group have resulted in a reclassification of the group with An. funestus, An. aruni, An.

\footnotetext{
* Correspondence: maureenc@nicd.ac.za

'Vector Control Reference Unit, National Institute for Communicable Diseases, National Health Laboratory Service, Johannesburg, South Africa Full list of author information is available at the end of the article
}

parensis, An. confusus and An. vaneedeni being grouped together as members of the "An. funestus subgroup", $A n$. rivulorum, An. rivulorum-like, An. brucei and An. fuscivenosus forming their own subgroup, and $A n$. leesoni being classified with the Asian Anopheles minimus subgroup [3]. Recently, a new species has been described from Malawi, An. funestus-like [4], and this species falls within the An. funestus subgroup.

In addition to the 10 species mentioned above, there are closely related species that are not included in the group because of morphological differences in the adult females - Anopheles longipalpis being one of these [1]. These differences are very subtle and the probability of 
confusing this species with the vector An. funestus is very high. To further complicate the issue, molecular studies on this taxon have revealed at least two cryptic species, one from South Africa (Type A) and the other from Zambia (Type C) [5].

The adult biology of An. longipalpis has not been well studied despite the fact that it is widely distributed in eastern and southern Africa, from Sudan to Angola and South Africa $[1,2,6]$. Smith $[7,8]$ reported human feeding behaviour in An. longipalpis from indoor and outdoor collections on Ukara Island in Lake Victoria and outdoors in Tanzania. Adugna and Petros [9] also found An. longipalpis specimens containing human blood meals from their collections in Ethiopia. Recently, Kent et al [10] reported that although the species is found in large numbers resting indoors in sympatry with An. funestus and Anopheles arabiensis in Zambia, it is predominantly zoophilic with only a small number feeding on humans. The species has never been implicated as a vector of malaria or involved in malaria transmission $[1,2,10,11]$. However, it may be a secondary vector of malaria in areas of sufficiently high endemicity and densities of this species [10].

Although adult An. longipalpis are morphologically characterized by maxillary palpal length and pale basal and apical bands spanning the hind tarsal joints $[1,2]$, this species can be misidentified as An. funestus due to similarities of wing venation, palpal banding patterns and small body size. Currently, there are two identification methods for field-collected specimens of An. longipalpis. The first method, suggested by Kent et al [10], is to identify An. longipalpis using the species-specific PCR assay for the An. funestus group from the internal transcribed spacer (ITS) region of the rDNA [11]. The assay produces two diagnostic fragments from An. longipalpis type $\mathrm{C}$ which correlates with $A n$. parensis and An. vaneedeni due to sequence similarity among these species. However, this analysis would lead to misidentification with hybrids of An. parensis and An. vaneedeni, although these have never been found in nature. The second method is a species-specific PCR assay for An. longipalpis from the ITS2 region developed by Koekemoer et al [5], but this is not specific when other members of the An. funestus group are included such as An. funestus, An. funestus-like and An. parensis. Hence, this study proposes a new method for the molecular identification of An. longipalpis type $\mathrm{C}$ and six members of the An. funestus group using a Restriction Fragment Length Polymorphism (RFLP) that is more accurate and efficient than currently used methods.

\section{Methods}

\section{Mosquito samples collection and identification}

Mosquitoes were collected from Malawi, Mozambique, South Africa and Zambia (Table 1). There were eleven An. longipalpis type $\mathrm{C}$ specimen available. Initially, specimens were identified morphologically using the keys of Gillies and Coetzee [2]. All DNA samples were extracted from either single mosquitoes or available parts of mosquitoes using the Ballinger-Crabtree protocol [12] except An. longipalpis where the method of Collins et al [13] was used. The DNA templates were resuspended in TE buffer at volumes between $50 \mu \mathrm{L}$ and $100 \mu \mathrm{L}$. All DNA templates were identified using the method of Spillings et al [4] for An. funestus-like and the method of Koekemoer et al [11] for the rest of the species.

\section{Restriction fragment length polymorphism (RFLP) assay}

Although the method of Spillings et al [4] can be carried out simultaneously with the method of Koekemoer et al [11], they recommend that these assays be carried out separately because the An. funestus-like $390 \mathrm{bp}$ fragment of Spillings et al [4] is close to the An. rivulorum $411 \mathrm{bp}$ fragment of Koekemoer et al [11]. The procedure in the present study simultaneously carried out both these methods $[4,11]$ with minor modifications.

Table 1 List of species, localities and numbers used

\begin{tabular}{|c|c|c|c|c|}
\hline \multirow[t]{2}{*}{ Species } & \multicolumn{2}{|r|}{ Localities } & \multirow[t]{2}{*}{ Numbers used } & \multirow[t]{2}{*}{ Tota } \\
\hline & Country & Village & & \\
\hline \multirow[t]{2}{*}{ An. funestus } & Mozambique & Chibuto $\left(24^{\circ} 40^{\prime} \mathrm{S}, 33^{\circ} 33^{\prime} \mathrm{E}\right)$ & 3 & 5 \\
\hline & Malawi & Karonga $\left(10^{\circ} 19^{\prime} \mathrm{S}, 34^{\circ} 08^{\prime} \mathrm{E}\right)$ & 2 & \\
\hline An. funestus-like & Malawi & Karonga $\left(10^{\circ} 19^{\prime} \mathrm{S}, 34^{\circ} 08^{\prime} \mathrm{E}\right)$ & 5 & 5 \\
\hline An. leesoni & South Africa & Komatipoort $\left(25^{\circ} 26^{\prime} \mathrm{S}, 31^{\circ} 57^{\prime} \mathrm{E}\right)$ & 3 & 3 \\
\hline An. longipalpis type $C$ & Zambia & Macha $\left(16^{\circ} 46^{\prime} \mathrm{S}, 26^{\circ} 94^{\prime} \mathrm{E}\right)$ & 11 & 11 \\
\hline An. parensis & South Africa & Mamfene $\left(27^{\circ} 23^{\prime} \mathrm{S}, 32^{\circ} 12^{\prime} \mathrm{E}\right)$ & 5 & 5 \\
\hline \multirow[t]{2}{*}{ An. rivulorum } & South Africa & Komatipoort $\left(25^{\circ} 26^{\prime} \mathrm{S}, 31^{\circ} 57^{\prime} \mathrm{E}\right)$ & 5 & 5 \\
\hline & & Giyani area $\left(23^{\circ} 15^{\prime} \mathrm{S}, 30^{\circ} 47^{\prime} \mathrm{E}\right)$ & 7 & \\
\hline \multirow[t]{3}{*}{ An. vaneedeni } & South Africa & Komatipoort area $\left(25^{\circ} 26^{\prime} \mathrm{S}, 31^{\circ} 57^{\prime} \mathrm{E}\right)$ & 28 & 46 \\
\hline & & Mamfene area $\left(27^{\circ} 23^{\prime} \mathrm{S}, 32^{\circ} 12^{\prime} \mathrm{E}\right)$ & 10 & \\
\hline & & Ndumu $\left(27^{\circ} 02^{\prime} S, 32^{\circ} 19^{\prime} \mathrm{E}\right)$ & 1 & \\
\hline
\end{tabular}


Table 2 Sizes of the DNA fragments after PCR-RFLP for the An. funestus group and An. longipalpis

\begin{tabular}{cccc}
\hline Species & PCR product sizes (bp) & DNA fragment length after digestion with EcoRI (bp) \\
\hline An. funestus & 505 & 505 & 15 \\
An. funestus-like & 390 (approx.) & 375 & \\
An. leesoni & 146 & 146 & 252 \\
An. Longipalpis type C & 587,252 & 376 & 211 \\
An. parensis & 252 & 411 \\
An. rivulorum & 411 & 587 \\
An. vaneedeni & 587 & & \\
\hline
\end{tabular}

The $\mathrm{G}^{\wedge} \mathrm{AATTC}$ restriction site for EcoRI enzyme lies within the An. funestus-like and An. longipalpis type C fragments amplified by the methods of Spillings et al [4] and Koekemoer et al [11] respectively. The restriction enzyme digested only the fragments for An. funestus-like and An. longipalpis type $\mathrm{C}$ directly. A total volume of 25 $\mu \mathrm{L}$ for each PCR reaction contained $1 \mu \mathrm{L}$ of the genomic DNA of an individual mosquito, $1 \times$ PCR Buffer, $0.2 \mathrm{mM}$ of each dNTP, $0.26 \mathrm{pM}(0.2 \mu \mathrm{M}$ of MalaFB primer) of each primer, and 1 unit of Taq DNA polymerase. The PCR cycling conditions were as follows: a 2 minute $94^{\circ} \mathrm{C}$ followed by 35 cycles of 30 seconds at $94^{\circ} \mathrm{C}, 30$ seconds at $50^{\circ} \mathrm{C}$ and 40 seconds at $72^{\circ} \mathrm{C}$; there was a final extension step of 10 minutes at $72^{\circ} \mathrm{C}$. After amplification, 1 unit of EcoRI in $1 \times$ buffer EcoRI (Roche Diagnostic) was added to the PCR reactions and digestion carried out at $37^{\circ} \mathrm{C}$ for a minimum of three hours. Digested fragments were electrophoresed through an ethidium bromide $2.5 \%$ agarose gel and photographed under ultraviolet light illumination using a gel imaging system.

\section{Results}

The PCR-RFLP assay for the six members of the $A n$. funestus group and An. longipalpis type C, resulted in different sizes of DNA fragments as recorded in Table 2. Two fragments (252 bp and $587 \mathrm{bp}$ ) of $A n$. longipalpis type $\mathrm{C}$ were amplified after the species- specific An. funestus group PCR. The restriction enzyme digested only the fragments for An. funestus-like and An. longipalpis type $C$. There were restriction sites at position 375 in An. funestus-like and at position 376 in the large fragment of An. longipalpis type C (Figure 1). Although the restriction site was identical to the DNA sequence of $A n$. parensis, the specific fragment was not involved in the primer design for the method of Koekemoer et al [11]. The digestion profiles for An. funestuslike and $A n$. longipalpis type $C$ were characterized by two fragments, $375 \mathrm{bp}$ and $15 \mathrm{bp}$ and three fragments, $211 \mathrm{bp}, 252 \mathrm{bp}$ and $376 \mathrm{bp}$ respectively. However, the length of fragment for An. funestus-like after PCR amplification was not accurate due to approximate length for the species in Spillings et al [4] and not available for the full alignment of DNA sequences in GenBank. Figure 2 shows DNA bands for the six members of the An. funestus group and An. longipalpis type C produced after PCR amplification and restriction enzyme digestion.

\section{Discussion}

The assay for the An. funestus group and An. longipalpis type $\mathrm{C}$ used in this study requires one PCR and one RFLP step instead of the three PCR steps currently needed. This will save time and reagents when routinely identifying collections of wild mosquitoes belonging to this important group. All seven species were easily separated on a $2.5 \%$

\begin{tabular}{|c|c|c|c|}
\hline & 357 & $\mathbf{v}$ & 406 \\
\hline An. funestus & \multicolumn{3}{|c|}{ TACGTTGTGAAACATGGGGAAATTCAATCGAAAACCTCTTTGATGTCCAA } \\
\hline An. funestus-like & \multicolumn{3}{|c|}{$\ldots \ldots \ldots$ АА } \\
\hline An. leesoni & \multicolumn{3}{|c|}{$--\ldots$ ACC ...G.T.CCC ..---- . GC.T.CT .A.C.CAG.C.AGT.G. } \\
\hline An. longipalpis & \multicolumn{3}{|c|}{ 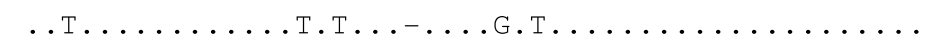 } \\
\hline An. parensis & \multicolumn{3}{|c|}{$\ldots$ Т...... } \\
\hline An. rivulorum & \multicolumn{3}{|c|}{$--\ldots$. CC ... . GCCCC ..-----..G---.CCTA.A.CAG .. TGT.GG } \\
\hline An. vaneedeni & & & \\
\hline
\end{tabular}

Figure 1 Alignment from $3^{\prime}$ to $5^{\prime}$ end of the ITS2 region of the rDNA sequenced by Koekemoer et al [5]for An. longipalpis type C, Spillings et al [4] for An. funestus-like and Koekemoer et al [11]for the rest of the species. Dots represent identity with respect to the An. funestus sequences. Dashes represent gaps in sequences. The black triangle indicates the restriction site of the EcoRl enzyme (G^AATTC) in An. funestus-like and An. longipalpis type C. The amplification for An. parensis after PCR-RFLP was not included in these sites. 


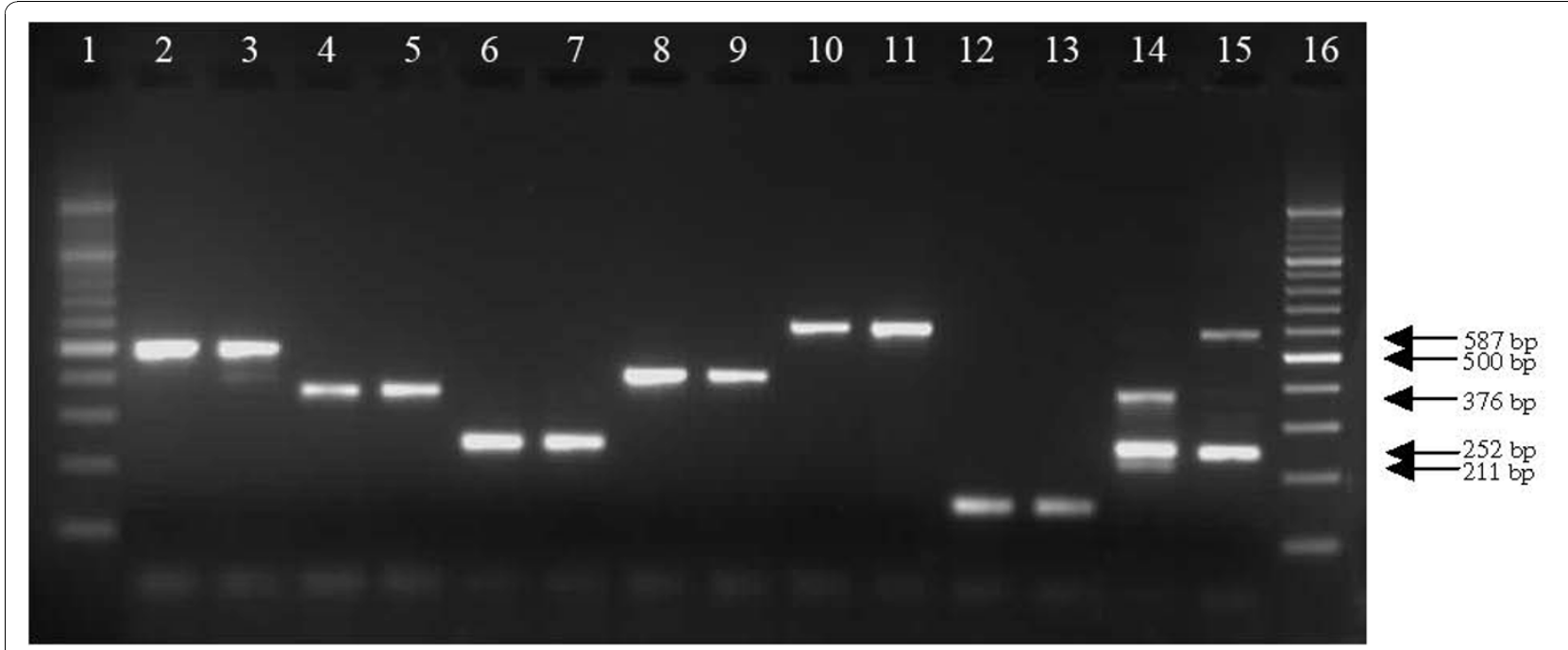

Figure 2 An ethidium bromide stained 2.5\% agarose gel showing the DNA bands after PCR-RFLP. Lanes 1 and $16=100 \mathrm{bp}$ molecular marker; lanes 2 and $3=$ An. funestus; lanes 4 and $5=$ An. funestus-like; lanes 6 and $7=$ An. parensis; lanes 8 and $9=$ An. rivulorum; lanes 10 and $11=$ An. vaneedeni; lanes 12 and $13=$ An. leesoni; lane $14=$ An. longipalpis type C; lane $15=1: 1$ An. parensis and An. vaneedeni mixed DNAs.

ethidium bromide agarose gel even though the digested amplicons of An. longipalpis type C (252 bp and $211 \mathrm{bp}$ ), An. funestus-like (375 bp) and An. rivulorum (411 bp) are close to each other. However, individuals that do not amplify at all may need additional processing using the An. longipalpis type A primers [5].

Koekemoer et al [5] reported that sequence analysis of the ITS2 region in An. longipalpis type $\mathrm{C}$ is similar to the sequences of $A n$. parensis and An. vaneedeni in the An. funestus subgroup. These three species are almost identical at the adult stage with only a few minor differences $[1,2]$. However, An. longipalpis was not included in the An. funestus group because of its very different larval morphology [1,3]. Koekemoer et al [5] indicated that it might be due to extensive evolutionary divergence and the sequence differentiation for An. longipalpis type A, which is close to Anopheles pampanai and Anopheles varuna in the Asian An. minimus group based on the DNA analysis of the ITS2 region. They suggested that An. longipalpis type $\mathrm{C}$ should be placed in the An. funestus group supported by sequence similarity to both An. parensis and An. vaneedeni. Furthermore, Kent et al. [10] also reported that the fragments diagnostic for $A n$. longipalpis type $C$ were the same as the diagnostic amplicons for a hypothetical An. parensis/An. vaneedeni hybrid although no such hybrids have been recorded in nature. However, further investigation of An. longipalpis is still required with more field specimens.

\section{Conclusion}

The application of the method described is expected to greatly improve the efficiency of large-scale analysis of field-collected samples of the An. funestus group and An. longipalpis type $\mathrm{C}$.

\section{Acknowledgements}

This work was supported by the Department of Science and Technology, National Research Foundation Research Chair Initiative grant to MC. We thank the following for assistance with field collections or supply of specimens: A. Mabuza (Department of Health, Mpumalanga Province, South Africa); K. Hargreaves (Department of Health, KwaZulu/Natal Province, South Africa); T. Ledwaba (Department of Health, Limpopo Province, South Africa) and Prof R.H. Hunt (University of the Witwatersrand).

\section{Conflict of interests}

The authors declare that they have no competing interests.

\section{Author details}

${ }^{1}$ Vector Control Reference Unit, National Institute for Communicable Diseases, National Health Laboratory Service, Johannesburg, South Africa. ${ }^{2}$ Malaria Entomology Research Unit, School of Pathology, Faculty of Health Sciences, University of the Witwatersrand, Johannesburg, South Africa.

\section{Authors' contributions}

KSC designed the study, developed the new RFLP assay and drafted the manuscript. MC and LLK assisted with analysis of the data and helped draft the manuscript.

All authors have read and approved the final manuscript.

Received: 18 June 2010 Accepted: 8 November 2010

Published: 8 November 2010

\section{References}

1. Gillies MT, de Meillon B: The Anophelinae of Africa South of the Sahara. Publications of the South African Institute for Medical Research, Johannesburg, South Africa; 1968.

2. Gillies MT, Coetzee M: A Supplement to the Anophelinae of Africa South of the Sahara (Afrotropical Region). Publications of the South African Institute for Medical Research, Johannesburg, South Africa; 1987.

3. Harbach RE: The classification of genus Anopheles (Diptera: Culicidae): a working hypothesis of phylogenetic relationships. Bull Entomol Res 2004, 94:537-553. 
4. Spillings BL, Brooke BD, Koekemoer LL, Chiphwanya J, Coetzee M, Hunt RH: A new species concealed by Anopheles funestus Giles, the major malaria vector in Africa. Am J Trop Med Hyg 2009, 81:510-515.

5. Koekemoer LL, Misiani EA, Hunt RH, Kent RJ, Norris DE, Coetzee M: Cryptic species within Anopheles longipalpis from southern Africa and phylogenetic comparison with members of the An. funestus group. Bull Entomol Res 2009, 99:41-49.

6. White GB: Confirmation that Anopheles longipalpis (Theobald) and Anopheles confusus Evans and Leeson occur in Ethiopia. Mos Syst 1972, 4:131-132.

7. Smith A: The transmission of Bancroftian filariasis on Ukara island, Tanganyika. I. A geographical and ecological description of the island with an annotated list of mosquitoes and other arthropods of medical importance. Bull Entomol Res 1955, 46:419-436.

8. Smith A: Observations on the man-biting habits of some mosquitoes in the South Pare area of Tanganyika. East Africa Med J 1961, 38:246.

9. Adugna N, Petros B: Determination of the human blood index of some anopheline mosquitoes by using ELISA. Ethiopian Med J 1996, 34:1-10.

10. Kent RJ, Coetzee M, Mharakurwa S, Norris DE: Feeding and indoor resting behavior of the mosquito Anopheles longipalpis in an area of hyperendemic malaria transmission in southern Zambia. Med Vet Entomol 2006, 20:459-463.

11. Koekemoer LL, Kamau L, Hunt RH, Coetzee M: A cocktail polymerase chain reaction assay to identify members of the Anopheles funestus (Diptera: Culicidae) group. Am J Trop Med Hyg 2002, 66:804-811.

12. Ballinger-Crabtree ME, Black WC, Miller BR: Use of genetic polymorphisms detected by the Random-Amplified Polymorphic DNA Polymerase Chain Reaction (RAPD-PCR) for differentiation and identification of Aedes aegypti subspecies and populations. Am J Trop Med Hyg 1992, 47:893-901.

13. Collins FH, Mendez MA, Rasmussen MO, Meheffey PC, Besansky NJ, Finnerty V: A ribosomal RNA gene probes differentiates members of the Anopheles gambiae complex. Am J Trop Med Hyg 1987, 37:37-41.

doi:10.1186/1475-2875-9-316

Cite this article as: Choi et al: Simultaneous identification of the Anopheles funestus group and Anopheles longipalpis type C by PCRRFLP. Malaria Journal 2010 9:316.

\section{Submit your next manuscript to BioMed Central and take full advantage of:}

- Convenient online submission

- Thorough peer review

- No space constraints or color figure charges

- Immediate publication on acceptance

- Inclusion in PubMed, CAS, Scopus and Google Scholar

- Research which is freely available for redistribution

Submit your manuscript at www.biomedcentral.com/submit
Biomed Central 